\title{
ANÁLISIS DE LA MOTIVACIÓN EXTRÍNSECA Y LA RETROALIMENTACIÓN AFECTIVA EN LA INTERACCIÓN PROFESOR-ALUMNO
}

\author{
Valeria García Corona, Edgardo Ruiz Carrillo, José Luis Cruz González y Cristina Gómez Aguirre \\ Universidad Nacional Autónoma de México \\ México
}

\begin{abstract}
RESUMEN
El objetivo de la presente investigación es identificar y analizar las categorías motivacionales del alumno y profesor que se presentan con mayor incidencia para interpretar el contenido de las mismas. La pregunta es cómo incide la retroalimentación afectiva en la motivación del alumno, proponiendo como hipótesis que la retroalimentación afectiva incide en la motivación y participación del alumno. El tipo de diseño es observacional, de tipo transaccional idiográfico multidimensional. La muestra fue no probabilística y por conveniencia $(n=32)$. Se videograbó un grupo de medicina, se transcribió y codificó la clase con base en el instrumento de categorías motivacionales. Se obtuvieron frecuencias e índices de cada una de ellas; además se realizó un análisis del discurso. Se encontró que la retroalimentación afectiva que el profesor ofrece fomenta la motivación del alumno, es decir, apreciar, reconocer y animar al alumno a contestar preguntas abiertas o directas.
\end{abstract}

Palabras Clave:

discurso, interacción profesor-alumno, motivación, participación, retroalimentación.

\section{ANALYSIS OF EXTRINSIC MOTIVATION AND AFFECTIVE FEEDBACK IN TEACHER-STUDENT INTERACTION}

\begin{abstract}
The objective of this research was to identify and analyze the motivational categories of the student and teacher that occur with the highest incidence to interpret their content. The question is how affective feedback affects student motivation, proposing as a hypothesis that affective feedback affects student motivation and participation. The type of design is observational, multidimensional idiographic transactional type. The sample was non-probabilistic and for convenience $(n=32)$. A medical group was videotaped, the class was transcribed and coded based on the motivational categories instrument. Frequencies and percentages of each one of them were obtained, in addition a discourse analysis was carried out. It was found that the affective feedback that the teacher offers fosters student motivation, that is, appreciating, acknowledging and encouraging the student to answer open or direct questions.
\end{abstract}

Keywords:

discourse, teacher-student interaction, motivation, participation, feedback.

Bitácora del Artículo:

| Recibido: 30 de Junio de 2021 | Aceptado: 30 noviembre de 2021 | Publicado en línea: Enero - Junio de 2022 | 
Artículo Empírico |Análisis de la motivación extrínseca y la retroalimentación...| García-Corona, et al. | e08012022376

\title{
Autoría y Derechos de Propiedad Intelectual
}

\author{
ANÁLISIS DE LA MOTIVACIÓN EXTRIINSECA Y LA RETROALIMENTACIÓN \\ AFECTIVA EN LA INTERACCIÓN PROFESOR-ALUMNO
}

\author{
Valeria García Corona, Edgardo Ruiz Carrillo, José Luis Cruz González y Cristina Gómez Aguirre \\ Universidad Nacional Autónoma de México \\ México
}

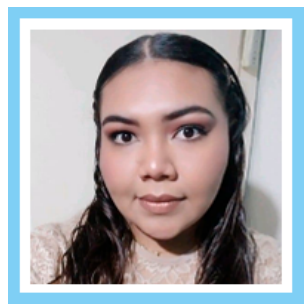

Valeria García Corona

FES- Iztacala, UNAM

Correo: valeriacorona03@outlook.com

Pasante de la carrera en Psicología generación: 2016-2019. (Universidad Nacional Autónoma de México. Facultad de Estudios Superiores Iztacala). Becaria de investigación/colaborador en el Programa de Apoyo a Proyectos para Innovar y Mejorar la Educación (PAPIME) con clave PE301620 enfocado en las estrategias de enseñanza-aprendizaje en el ámbito educativo. Asistente y presentadora en Congreso Nacional e Internacional de Psicología Educativa.

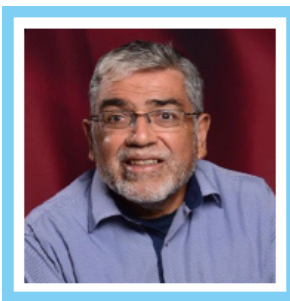

Edgardo Ruiz Carrillo FES- Iztacala, UNAM Correo:edgardo@unam.mx

Licenciatura en Psicología Facultad de Psicología. U.N.A.M. octubre de 1981. Maestría en Psicobiología. U.N.A.M. Facultad de Psicología, 1986. Doctorado en Investigación Psicológica, Universidad Iberoamericana, 2004. Distinción como Investigador Nacional Nivel 1 (Sistema Nacional de Investigadores Conacyt) durante el periodo del 01 de enero de 2018 al 31 de diciembre de 2021. Ratificación para seguir siendo del Padrón de Tutores del Plan de Estudio de Doctorado en Psicología de la UNAM.

\section{CONTRIBUCIÓN DE LOS Autores}

Valeria García: documentación científica, grabación y recopilación de datos, codificación, análisis de resultados, y elaboración de discusión y conclusiones. I Edgardo Ruíz: Documentación científica, análisis de resultados y elaboración de conclusiones y discusión. I José Cruz: Grabación y recopilación de datos, codificación, análisis de resultados y elaboración de conclusiones y discusión. | Cristina Gómez: Documentación científica, codificación, análisis de resultados y elaboración de conclusiones y discusión.

\section{AgRAdECIMIENTOS}

La investigación fue apoyada por el Programa de Apoyo a Proyectos para Innovar y Mejorar la Educación (PAPIME) con clave PE301620.

\section{Datos de Filiación de los Autores}

Facultad de Estudios Superiores Iztacala, UNAM

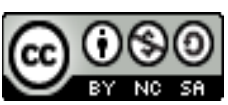

Copyright: @ 2022 García-Corona, V., Ruíz-Carrillo, E. Cruz-González, J.L. \& Gómez-Aguirre, C.

Este es un artículo de acceso abierto distribuido bajo los términos de la licencia Creative Commons Reconocimiento-NoComercial 4.0 Internacional, por lo que su contenido gráfico y escrito se puede compartir, copiar y redistribuir total o parcialmente sin necesidad de permiso expreso de sus autoras con la única condición de que no se puede usar con fines directamente comerciales y los términos legales de cualquier trabajo derivado deben ser los mismos que se expresan en la presente declaración. La única condición es que se cite la fuente con referencia a la Revista Digital Internacional de Psicología y Ciencia Social y a sus autoras. 


\section{TABLA DE CONTENIDO}

MÉTODO

Participantes, 5

Materiales, 5

Consideraciones éticas, 6

Procedimiento

Mediciones, 6

Análisis estadísticos, 6

RESULTADOS

DISCUSIÓN

CONCLUSIONES 
e acuerdo con Prados-Gallardo, Cubero-Pérez y De la Mata (2010), el constructivismo es la perspectiva que estudia la construcción conjunta de significados que se da por medio de la interacción e intercambio de información entre los participantes dentro del aula educativa. Es una teoría que entiende cómo las personas construyen nuevos conocimientos, dependiendo de las condiciones culturales y sociales en las que se desarrollan y aprenden (Ortiz, 2015). Por tanto, una persona construye nuevos conocimientos cuando hace una co-construcción de significados del contexto en que está aprendiendo; esto se logrará en el salón de clases por medio del papel que desarrollan el alumno y el profesor, y las interacciones que se establezcan entre ambos.

La psicología educativa busca analizar las interacciones entre profesor-alumno, así como desarrollar y mejorar las capacidades de los mismos, con la finalidad de que el proceso de enseñanza-aprendizaje sea exitoso (Pérez, 2019). Dicho proceso se logra por el intercambio de conocimientos entre profesor-alumno dentro del salón de clases. Cada uno desempeña una función y conductas que pueden ser o no motivantes para involucrarse en el proceso; las habilidades del profesor repercuten en el aprendizaje de los alumnos, por lo que es importante que el primero desarrolle y mejores sus habilidades de enseñanza dentro del aula, para fomentar la motivación del alumno y, a su vez, su participación activa del mismo.

Un elemento fundamental para el aprendizaje de los alumnos es la motivación; este concepto es visto como la fuerza interna que tiene una persona para moverse, actuar y lograr un objetivo claro, y mantenerla a lo largo del tiempo (García y Cruz, 2016). Estos mismos autores mencionan que cuando un alumno está motivado se involucra de manera activa en las clases y se compromete a adquirir los conocimientos que el profesor transmite.

Algunos autores clasifican la motivación en dos grandes categorías: 1) intrínseca y 2) extrínseca. Ryan, Connell y Deci (1985, citado en García y Cruz, 2016) explican que la motivación intrínseca es la satisfacción promovida por la actividad que se está llevando a cabo, mientras que la extrínseca tiene una intencionalidad instrumental, busca un incentivo ajeno a dicha actividad y/o un reconocimiento físico o social (Echeverría, Pacenza y Urquijo, 2011; Héctor, 2012; Seder, Castell y Moreno, 2018). Entonces, la primera busca una satisfacción individual por aprender y la segunda una satisfacción externa, en donde el otro reconozca lo que él mismo está haciendo.
Si un alumno hace lo que el profesor espera que haga es porque el primero se encuentra motivado (García y Cruz, 2016). Valenzuela et al. (2018) indican que el estudiante motivado intrínsecamente va a verse activo, lleno de energía, estudia fuera del curso, siente placer y necesidad por aprender, mientras que el alumno motivado extrínsecamente va a atender las preguntas que le hacen y va a cuestionar para que alguien reconozca su participación.

Es importante que el profesor esté motivado porque ayuda a que el alumno también lo esté y logre sus objetivos (Alemán-Marichal et al., 2018). Una función del profesor que motiva al alumno es relacionar los contenidos previos con los nuevos, e incluso con otros temas y materias, observando una relación entre éstos y no un aislamiento; esto es significativo para el estudiante porque cumple con sus necesidades formativas (López y Villalobos, 2010). Del mismo modo, es importante que aborde los temas de lo simple a lo complejo, y se tome el tiempo necesario para que los estudiantes logren comprenderlos sin saturarlos de información, a la vez de no subestimar sus capacidades y habilidades para aprender (Polanco, 2005). Por tanto, el discurso que el profesor utiliza se refleja en la motivación del alumno y en sus participaciones; de esta manera los estudiantes co-construyen los aprendizajes y los expresan en sus aportaciones, esperando que el profesor les reconozca su saber.

A partir de esto, Boza-Carreño y De la O Toscano (2012) mencionan que los estudiantes deben sentirse con libertad para expresar sus dudas y puntos de vista ante cualquier tema, pues el profesor no debe poner barreras para explicar o retroalimentar lo dicho; es por ello que este último debe contar con una preparación adecuada sobre estrategias en el aula y solución de problemas.

Aunado a esto, Ruiz, Méndez y Cruz (2017) sugieren que la retroalimentación (feedback) que el profesor ofrece es esencial en la co-construcción de significados, aprendizaje y motivación del alumno, porque si éste retroalimenta haciendo correcciones, reformulando, complementando y enriqueciendo las respuestas de los alumnos, permite que haya una negociación de significados, además de alentar y reconocer el esfuerzo del alumno por participar.

Por su parte, Barrios (2008) y Mayoral y Castelló (2015) mencionan que la retroalimentación se clasifica en 1) cognitiva y 2) afectiva. En la primera se analizan los conocimientos que el alumno tiene respecto al tema de clase, el profesor corrige la respuesta del alumno para que él mismo analice su respuesta y la corrija, mientras que en la segunda el profesor aprueba la participación del estudiante proporcionando un reconocimiento ver- 
bal, lo que ocasiona que el alumno se sienta seguro y confiado de participar.

Además, González (2005) agrega que la retroalimentación afectiva motiva que el estudiante se involucre en el proceso de aprendizaje, es decir, el alumno participe en clase cuando el profesor reconozca su esfuerzo por hacerlo, de manera que participará activamente, responderá correcto a preguntas que se le formulen y hará comentarios de manera voluntaria, mejorando sus calificaciones $y$, de este modo, evitará el fracaso escolar (Héctor, 2012).

La retroalimentación afectiva que el profesor expresa puede ser mediante elogios, y éstos desempeñan un papel determinante en la participación y motivación del alumno en la clase (Lundgren y Lökholm, 2006). Jiménez (2014) complementa diciendo que el elogio es una herramienta que el profesor utiliza para reconocer los méritos o cualidades del alumno, y que dicho elogio es dirigido al esfuerzo, a la autorregulación, al compromiso o a los procesos relacionados con la tarea. Permite al estudiante asumir un papel activo en el aprendizaje respecto a la información proporcionada por el profesor; cuando el estudiante se motiva, logra un compromiso con los objetivos de aprendizaje para el mejoramiento de la autoeficacia o entendimiento de la tarea. Sin embargo, ninguno de los autores menciona la constancia y variabilidad con que se debe efectuar la retroalimentación afectiva para que sea efectiva en la motivación del estudiante.

Black y Wiliam (2004) y Bruno y Santos (2010) afirman que el éxito del feedback depende del conocimiento de las dificultades, habilidades y personalidad que tenga el profesor de cada uno de sus estudiantes en situaciones particulares. Esto permite al profesor ahondar en el proceso del estudiante para hacer cambios y planes de acción para el mejoramiento del mismo; por tanto, el proceso de feedback debe ser: 1) inmediato; 2) continuo, y 3) relevante (López, 2010).

En este estudio se retomara la clasificación de retroalimentación afectiva que proponen Barrios (2008) y Mayoral y Castelló (2015) debido a la importancia que tiene en la motivación, participación y el apoyo emocional del alumno, puesto que la retroalimentación afectiva contribuye a generar y/o incrementar la confianza que tiene el alumno respecto a los conocimientos que ha adquirido, y a su vez poder compartirlos con la clase para juntos co-construir nuevos aprendizajes. Sin embargo, los estudios retomados no han indagado la relación entre la retroalimentación afectiva y la motivación extrínseca, vista como la participación oral dentro de contextos educativos.

Por tanto, el objetivo es identificar las categorías motivacionales del profesor, tal como el discurso, preguntas que plantea y la retroalimentación afectiva que hace, así como analizar cuáles se presentan con mayor incidencia e interpretar el contenido de las mismas, además de observar si esto se refleja en la motivación extrínseca del alumno, es decir, en su participación dentro de una clase de medicina. Para este propósito, la pregunta de investigación que se formula es "¿Cómo incide la retroalimentación afectiva del profesor en la motivación extrínseca del alumno (participación)?", y la hipótesis de investigación que se plantea es que la retroalimentación afectiva sí incide en la motivación extrínseca del alumno, es decir, que cuando el profesor reconoce y alienta al alumno, éste participa de manera voluntaria.

\section{Método}

\section{Participantes}

Los participantes fueron 32 estudiantes (13 hombres y 19 mujeres) de entre 17 a 22 años de primer semestre de la carrera de Médico Cirujano, y una profesora de una Institución Pública de Educación Superior (IPES); todos los participantes son de nacionalidad mexicana. La selección fue mediante un muestreo no probabilístico por conveniencia.

Los criterios de inclusión para la profesora fueron: ser mayor de edad, tener al menos cinco años fungiendo como profesor de la asignatura y haber firmado el consentimiento informado; y los criterios de inclusión para los alumnos fueron: ser mayor de edad, ser estudiante del primer semestre de la carrera de Médico Cirujano en la IPES, estar inscrito con la profesora a cargo y haber firmado el consentimiento informado. El criterio de exclusión para todos fue que no quisieran participar y que no firmaran el consentimiento informado.

El tipo de diseño es observacional, de tipo transaccional idiográfico multidimensional, debido a que se efectuó sólo una sesión de observación cuya muestra se comporta como una unidad, y las categorías operacionales tienen más de una dimensión (Anguera, Blanco-Villaseñor, Losada y Portell, 2018).

\section{Materiales}

Se hizo una observación natural (Anguera, 2003) desarrollada en un contexto natural/habitual, que consiste en un procedimiento científico que evidencia la ocurrencia de conductas perceptibles para poder llevar un registro organizado y su análisis - tanto cualitativo como cuantitativo-; esto se efectúa mediante un instrumento adecuado y parámetros convenientes, posibilitando la detección de las relaciones de diverso orden existentes entre ellas y evaluándolas. Dichas conductas, por la es- 
pontaneidad o habitualidad con que ocurren, evidencian los elementos que es necesario destacar para lograr su objetivación adecuada.

Como herramientas para recuperar las conductas perceptibles se utilizaron una videocámara marca Canon modelo VIXIA HF-R50 y un micrófono externo SHURE VF83, con el objetivo de recabar información fidedigna.

\section{Consideraciones éticas}

El trabajo se realizó siguiendo las recomendaciones del Código Ético del Psicólogo de la Sociedad Mexicana de Psicología (2010), que indican trabajar con el consentimiento informado de los participantes, guardar su confidencialidad al usar seudónimos para evitar la identificación de la persona, e informarles del momento en que se estaba grabando, así como de su derecho a obtener la trascripción de los datos analizados. Estas consideraciones son rescatadas de los artículos 122 y 134 . Antes de videograbar la clase, se explicó al profesor y a los alumnos el objetivo de la investigación y cuál sería su participación en la misma. Además se les proporcionó el formato de consentimiento informado por escrito; si aceptaban participar debían fírmalo; se les puntualizó que sería confidencial y los datos recabados eran con fines académicos y científicos. Luego de grabar la clase, los discursos durante la misma fueron transcritos, registrando a su vez conductas y acciones verbales del grupo.

\section{Procedimiento}

Se contactó con el profesor y el grupo de licenciatura de Médico Cirujano; después de explicar los motivos de la investigación y efectuar la firma de consentimientos informados, se concretó una fecha para hacer la investigación. La clase se grabó el martes 19 de marzo de 2019, en un horario de 9 a 13 hrs. Durante la clase, uno de los investigadores se colocó en la esquina izquierda delantera del salón, a un costado del escritorio del profesor, para realizar la video filmación de la clase; se utilizó una videocámara con un micrófono externo. Luego se revisó la filmación; al no encontrar dificultades de video y audio, los discursos de la misma fueron transcritos, registrando a su vez conductas y acciones verbales del grupo.

Posteriormente se creó un sistema de categorías, elaborado a partir de la transcripción de la videograbación y en función del objetivo de estudio. Por último se calcularon frecuencias y porcentajes de las categorías motivacionales de los alumnos y el profesor para identificar las categorías que se presentan con mayor y menor incidencia, y luego se tomaron algunos frag- mentos discursivos del profesor y alumno para ejemplificar las categorías de interés, dar sentido, explicación e interpretación a éstas, y conocer cómo se presentaron durante la clase.

\section{Mediciones}

El estudio se orienta a conductas de naturaleza nominal o categórica en las que, de acuerdo con Anguera et al. (2018), los instrumentos de observación esencialmente son tres: 1) sistema de categorías; 2) formato de campo, y 3) combinación entre formato de campo y sistemas de categorías (Anguera y Blanco-Villaseñor, 2006; Anguera, Magnusson y Jonsson, 2007), por lo cual el instrumento de uso para la presente investigación corresponde a esta combinación (tabla 1).

De este modo, se identificaron actividades relacionadas a nuestras definiciones operacionales que coinciden con la motivación extrínseca del alumno, es decir, su participación en clase al contestar y plantear preguntas y hacer aportaciones. Así como las acciones que el profesor efectúa para que el alumno participe: explicar los contenidos, plantear preguntas y retroalimentación afectiva de Barrios (2008) y Mayoral y Castelló (2015).

El instrumento de categorías motivacionales se puso a discusión y valoración de investigadores con experiencia en el tema para aprobar su utilización en el presente estudio (Robles y Rojas, 2015). Se elaboraron listas de conductas y situaciones hasta llegar a la exhaustividad y la mutua exclusividad (E/ME) de las categorías, de manera que existiera una categoría para cada acción posible, y una acción posible para cada categoría de interés.

\section{Análisis estadísticos}

Una vez transcritas las grabaciones, se utilizó el software MAXQDA20 para hacer la codificación de las transcripciones y el análisis de las mismas. De acuerdo con Casasempere (2017), el software permite observar las frecuencias de las categorías motivacionales emitidas por el alumno y el profesor, lo que permitió identificar el orden en que se presentan las categorías; esto se hizo con la herramienta de codificación de datos, estableciendo la cantidad de veces que se presentan las categorías durante la clase. Después se elaboró un análisis del discurso en donde se retomaron algunos fragmentos discursivos del profesor y el alumno para ejemplificar las categorías de interés y explicar e interpretar cómo se presentaron durante la clase; el principal objetivo fue encontrar relación entre el texto y el contexto para entender cómo se construye el aprendizaje (Urra, Muñoz y Peña, 2013). 
Tabla 1. Categorías de motivación extrínseca en la interacción profesor-alumno con clave y definición.

\begin{tabular}{|c|c|c|c|}
\hline Participante & Categoría & Definición & Clave \\
\hline \multirow{7}{*}{ Alumno } & Pregunta al profesor & $\begin{array}{l}\text { El alumno formula una pregunta clara y directa } \\
\text { sobre el tema de la clase al profesor. }\end{array}$ & Prel \\
\hline & Pregunta a los alumnos & $\begin{array}{l}\text { El alumno formula una pregunta clara y abierta } \\
\text { sobre el tema de clase a sus compañeros. }\end{array}$ & PreG \\
\hline & Respuesta teórica dirigida & $\begin{array}{l}\text { El alumno responde de manera teórica o } \\
\text { conceptual a una pregunta que el profesor le ha } \\
\text { solicitado directamente. }\end{array}$ & Rteol \\
\hline & Respuesta teórica no dirigida & $\begin{array}{l}\text { El alumno responde de manera teórica o } \\
\text { conceptual a una pregunta que el profesor ha } \\
\text { solicitado abiertamente al grupo. }\end{array}$ & RteoG \\
\hline & Respuesta complementaria & $\begin{array}{l}\text { El alumno agrega información a la respuesta de } \\
\text { otro participante. }\end{array}$ & Rcomp \\
\hline & Respuesta reflexiva & $\begin{array}{l}\text { El alumno emplea su propia experiencia como } \\
\text { referente crítico. }\end{array}$ & Rrefl \\
\hline & Solicitud de participación & $\begin{array}{l}\text { El alumno levanta la mano para participar: } \\
\text { hacer una pregunta, responder una pregunta o } \\
\text { complementar una respuesta. }\end{array}$ & SolP \\
\hline \multirow{4}{*}{ Profesor } & Pregunta abierta & $\begin{array}{l}\text { El profesor formula una pregunta de sondeo } \\
\text { dirigida a todo el grupo. }\end{array}$ & PreAbP \\
\hline & Pregunta dirigida & $\begin{array}{l}\text { El profesor formula una pregunta de sondeo } \\
\text { dirigida a un alumno específicamente. }\end{array}$ & PreDiP \\
\hline & Descripción teórica & $\begin{array}{l}\text { El profesor explica los contenidos de la clase de } \\
\text { manera teórica o conceptual. }\end{array}$ & DesTePro \\
\hline & Descripción coloquial & $\begin{array}{l}\text { El profesor explica los contenidos de la clase con } \\
\text { ejemplos de la vida cotidiana. }\end{array}$ & DesCoPro \\
\hline \multirow{7}{*}{ Retroalimentación afectiva } & Asignación de participación & $\begin{array}{l}\text { El profesor asigna turno de participación a los } \\
\text { alumnos que levantan la mano. }\end{array}$ & ParPro \\
\hline & Reconocimiento & $\begin{array}{l}\text { El profesor manifiesta que está de acuerdo con la } \\
\text { respuesta u opinión del alumno y complementa su } \\
\text { respuesta. }\end{array}$ & RecPro \\
\hline & Apreciación & $\begin{array}{l}\text { El profesor admite la respuesta del alumno como } \\
\text { correcta y la repite. }\end{array}$ & AprePro \\
\hline & Comprensión & $\begin{array}{l}\text { El profesor entiende las dificultades que el alumno } \\
\text { experimenta para expresar sus conocimientos. }\end{array}$ & ComPro \\
\hline & Ánimo & El profesor alienta al estudiante para participar. & AniPro \\
\hline & Ofrecimiento & $\begin{array}{l}\text { El profesor se presta voluntariamente para volver a } \\
\text { explicar un contenido al estudiante. }\end{array}$ & OfrePro \\
\hline & Agradecimiento & $\begin{array}{l}\text { El profesor expresa gratitud al estudiante por su } \\
\text { respuesta. }\end{array}$ & AgraPro \\
\hline
\end{tabular}

Fuente: Barrios (2008) y Mayoral y Castelló (2015).

\section{Resultados}

A partir de la información obtenida de los videos de la clase y su transcripción, se hicieron dos tipos de análisis: cuantitativo y cualitativo. Para el primero se ob- tuvieron las frecuencias y porcentajes de las categorías motivacionales de los participantes, divididas en las del alumno (tabla 2, figura 1), y del profesor (tabla 3, figura 2); además se identificaron las categorías que se presentaron con mayor recurrencia; para el segundo análisis se 
tomaron fragmentos discursivos del profesor y del alumno para ejemplificar las categorías de interés y explicar

Tabla 2. Frecuencias y porcentajes de las categorías motivacionales del alumno.

\begin{tabular}{|c|c|c|c|c|}
\hline PARTICI & PANTE & Clave & FrECUENCIA & PORCENTAJI \\
\hline \multirow[t]{8}{*}{ Alumno } & & Prel & 6 & 3.35 \\
\hline & & PreG & 0 & 0 \\
\hline & & Rteol & 71 & 39.66 \\
\hline & & RteoG & 42 & 23.4 \\
\hline & & Rcomp & 26 & 14.52 \\
\hline & & Rrefl & 2 & 1.11 \\
\hline & & SolP & 32 & 17.87 \\
\hline & Total & 7 & 179 & 100 \\
\hline \multicolumn{5}{|l|}{50} \\
\hline \multirow{2}{*}{\multicolumn{5}{|c|}{$\begin{array}{l}40 \\
35\end{array}$}} \\
\hline & & & & \\
\hline \multicolumn{5}{|l|}{ 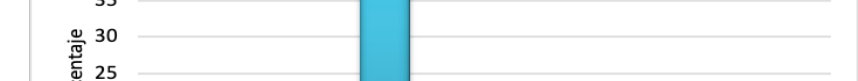 } \\
\hline \\
\hline \multicolumn{5}{|l|}{15} \\
\hline \multicolumn{5}{|l|}{$\begin{array}{r}10 \\
5\end{array}$} \\
\hline 0 & $\square$ & & & \\
\hline & & Rteol & og Rcomp & Solp \\
\hline
\end{tabular}

e interpretar cómo se presentaron durante la clase.

Tabla 3. Frecuencias y porcentajes de las categorías motivacionales del profesor..

\begin{tabular}{|cccc|}
\hline \multicolumn{1}{|c}{ Participante } & Clave & Frecuencia & Porcentaje \\
\hline Profesor & PreAbP & 47 & 13.12 \\
& PreDiP & 49 & 13.68 \\
& DesTePro & 54 & 15.08 \\
& DesCoPro & 28 & 7.82 \\
\hline $\begin{array}{l}\text { Retroalimentación } \\
\text { afectiva }\end{array}$ & ParPro & 32 & 8.93 \\
\hline & RecPro & 22 & 6.14 \\
\hline & AprePro & 57 & 15.92 \\
\hline & ComPro & 7 & 1.95 \\
\hline & AniPro & 49 & 13.68 \\
& OfrePro & 10 & 2.79 \\
\hline Total & AgraPro & 3 & 0.83 \\
\hline $\begin{array}{c}\text { Fuente: Elaboración Propia. } \\
\text { 11 }\end{array}$ & 358 & 100 \\
\hline
\end{tabular}

Fuente: Elaboración Propia.

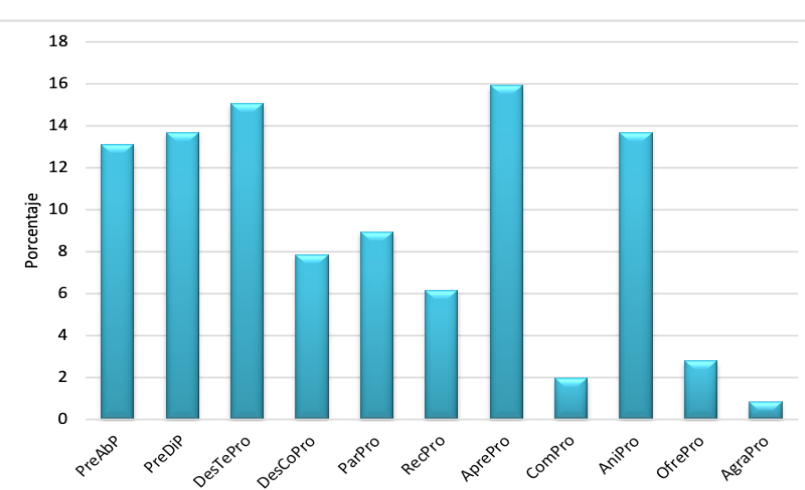

Figura 1. Porcentaje de las categorías motivacionales del alumno. Figura 2. Porcentaje de las categorías motivacionales del profesor.

En la tabla 2 se muestra una frecuencia total de 179 conductas, correspondientes a siete categorías motivacionales emitidas por el alumno. En la figura 1, estas frecuencias se expresan como porcentajes, donde se puede ver que la categoría que el alumno presentó de mayor porcentaje fue "Respuesta teórica no dirigida" (RteoG), con $39.66 \%$, y en segundo lugar "Respuesta teórica dirigida" (Rteol), con $23.46 \%$, mientras que las categorías que se presentaron en menor medida fueron "Respuesta reflexiva" (ReRrefl), con un índice de $1.11 \%$, y "Pregunta a los alumnos" (PreG), con presencia nula.

En la tabla 3 se observa un total de 358 conductas, correspondientes a 11 categorías motivacionales emitidas por el profesor. En la figura 2 se muestra la frecuencia de conductas como porcentajes, y en el gráfico de barras se observa que la categoría que el profesor presentó con mayor porcentaje fue "Apreciación" (AprePro), con $15.92 \%$, y enseguida la categoría "Descripción teórica" (DesTePro), con 15.08\%, y en tercer lugar las categorías "Ánimo" (AniPro) y "Pregunta directa" (PreDiP), con $13.68 \%$, mientras que las categorías con menor presencia fueron "Ofrecimiento" (OfrePro), con $2.79 \%$, seguida de "Comprensión" (ComPro), con 1.95\%, y "Agradecimiento", con 0.83\%.

Para el análisis cualitativo se tomarán algunos fragmentos discursivos del profesor y del alumno para ejemplificar las categorías presentes con mayor porcentaje (tabla 4), y luego se procederá a analizar, explicar e interpretar el contenido de las mismas, y si esto se refleja en el contexto en que se presenta el discurso, con la finalidad de dar una riqueza interpretativa.

En la tabla 4 se muestra que la categoría "Respuesta teórica dirigida" (Rteol) consiste en eventos donde el alumno respondió de manera teórica o conceptual a una pregunta, con base en el conocimiento previo que tenía, pero era muy breve en su respuesta. La participación que tuvo el alumno de medicina reflejó estar invo- 
Tabla 4. Descripción y ejemplo discursivo de algunas categorías motivacionales de alumno y maestro.

\begin{tabular}{|c|c|c|c|}
\hline Participante & Categoría & DEFINICIÓN & EJEMPLO DISCURSIVO \\
\hline & $\begin{array}{l}\text { Respuesta } \\
\text { teórica dirigida } \\
\text { (Reteol) }\end{array}$ & $\begin{array}{l}\text { El alumno responde } \\
\text { de manera teórica } \\
\text { o conceptual a una } \\
\text { pregunta que el } \\
\text { profesor le ha solicitado } \\
\text { directamente. }\end{array}$ & $\begin{array}{l}\text { "Y se presenta un lenguaje incoherente, agresividad, letargia, } \\
\text { estupor y vómito." }\end{array}$ \\
\hline
\end{tabular}

“En el entorno ambiental, bueno; también respecto al entorno es más predisponible que se lleve una persona a desarrollar alcoholismo en un entorno violento."

Alumno

Respuesta teórica no dirigida (ReteoG)

El alumno responde de manera teórica o conceptual a una pregunta que el profesor ha solicitado abiertamente al grupo.

\begin{tabular}{|l|l}
\hline & $\begin{array}{l}\text { Apreciación } \\
\text { (AprePro) }\end{array}$ \\
\hline Profesor & $\begin{array}{l}\text { Descripción } \\
\text { teórica } \\
\text { (DesTePro) }\end{array}$ \\
\hline & \\
\hline Ánimo (AniPro) \\
\hline $\begin{array}{l}\text { Pregunta directa } \\
\text { (PreDiP) }\end{array}$ \\
\hline
\end{tabular}

Fuente: Elaboración Propia.

lucrado en el tema de clase, respondió correctamente a los cuestionamientos que le hacía el profesor, pero no profundizó en ellas; el alumno construyó conocimientos de la clase y lo reflejó en sus respuestas, realizando una co-construcción de significados por medio de su discurso al grupo y a la clase. Caso contrario a la categoría "Respuesta teórica no dirigida" (RteoG), donde se evi-
“De los factores psicológicos y social, dice que las personas con baja autoestima podrían desarrollarlo, alcoholismo, pero y que lo desarrollan más si están muy presionadas por gente que los rodea."

"Se supone que se hacen unas preguntas y a las preguntas les dan cierta puntuación, y se divide ese puntaje en clasificación si es independiente, si sólo tiene consumo ocasional o si ya tiene un consumo que puede ser perjudicial, y bueno también este se puede determinar el nivel de riesgo y la enfermedad que puede tener, bueno eso fue lo que encontré."

Alumno: "Amor propio."

Profesor: "Amor propio, okey."

Alumno: "Incordinación".

Profesor: "Incoordinación, ajá."

Alumno: "Es una vía de escape."

Profesor: "Es una vía de escape."

“Y también tiene que ver con los neurotransmisores; si tenemos bloqueados los neurotransmisores con el alcohol no va a haber una respuesta motora; tiene que ver con reflejos, y entonces va a estar la pupila dilatada; no va a haber una respuesta muscular."

Alumno: "Tengo una duda, ¿es lo mismo tristeza que depresión?" Profesor: "Depresión tiene un componente etiológico a nivel de neurotransmisores; la tristeza es parte de su proceso de duelo; la depresión en una enfermedad, ¿sí? No son lo mismo."

"El jueves lo vimos; vamos, recuerden."

"A ver, inténtalo."

"Puedes recordarlo, inténtalo."

Alumno: "Un ambiente traumático también."

Profesor: "Tú mismo, ¿a qué se refiere un ambiente traumático?" Alumno: "Bueno, yo siento como que problemas en su familia, un trauma que haya tenido."

denció que el alumno contestó de manera voluntaria a una pregunta que el profesor formuló al grupo, dando una respuesta con base en el conocimiento previo que tenía, explicándolo de manera teórica y correcta.

La respuesta teórica no dirigida del alumno se caracteriza por ser profunda, detallada, clara y teórica de acuerdo con el tema que se abordaba, lo que reflejó que 
tenía conocimientos necesarios para responder, e hizo uso del material intelectual disponible durante la clase. A su vez, el estudiante hizo una co-construcción de significados ofrecidos por el profesor, y esto lo ayudó a externalizar sus conocimientos, haciendo uso de su propio acervo lingüístico aplicado al tema abordado, y por eso es que sus respuestas fueron largas, por lo que detallaron los conocimientos previos y actuales.

Por otro lado, en la categoría "Apreciación" (AprePro), emitida por el profesor, se observó que después de que el alumno contestó de manera teórica, el profesor reconoció la respuesta del alumno, es decir, utilizó palabras como "okey" y "ajá" para dar a conocer al alumno que la respuesta era correcta; en ocasiones el profesor repitió la respuesta que el alumno acaba de dar, lo que indica una manera de afirmar que había sido correcta.

La categoría "Descripción teórica" (DesTePro) se vio cuando el profesor explicó de manera clara, descriptiva y teórica la temática de la clase. Esta descripción resultó relevante cuando un alumno tenía una duda; el profesor fue el encargado de aclararla con base en lo escrito y se apoyó de elementos dentro de su experiencia o impresiones personales. Por ende, la explicación teórica del profesor tuvo un impacto en la comprensión del alumno; cuando el primero explicó de manera clara y teórica, se esperó que el alumno lograra entender los contenidos, lo cual permitiría que juntos realizaran una co-construcción del conocimiento.

Enseguida, la categoría "Ánimo" (AniPro) se caracterizó porque el profesor alentó al estudiante a participar por medio de preguntas abiertas o directas cuando a éste se le dificultó hablar y ampliar su respuesta; ante ello, el profesor actuó con frases que impulsaron al alumno a continuar hablando. El apoyo que el profesor ofreció a los alumnos resultó motivante para ellos, haciéndolos participar activamente y dar una respuesta con lo que ellos sabían, por lo que se infiere que cuando el profesor estimula a los estudiantes para que participen o amplíen sus conocimientos, es porque los considera capaces de complementar su respuesta y profundizar en ella.

Por último, la categoría "Pregunta directa" (PreDiP) se presentó cuando el profesor formuló una pregunta de sondeo dirigida específicamente a un alumno. El profesor buscó que el alumno ampliara y profundizara su respuesta para corroborar que tenía el conocimiento necesario del tema de clase. Por tanto, esta categoría se caracterizó por preguntas directas del profesor a los alumnos para que éstos profundizaran en sus respuestas, y quedara claro el conocimiento que se estaba construyendo en la clase.

\section{Discusión}

El objetivo de la presente investigación es identificar y analizar las categorías motivacionales del alumno y el profesor que se presentan con mayor incidencia, y luego interpretar el contenido de las mismas. Se encontró que el profesor utiliza un lenguaje teórico y conceptual (DesTePro) para explicar los contenidos de la clase a sus alumnos, lo cual es motivante para éstos; los alumnos replican dicho conocimiento cuando se les solicita contestar una pregunta o cuando hacen una participación voluntaria (Rteol y RteoG). De acuerdo con García y Cruz (2016), los profesores deben tener la habilidad de usar un vocabulario entendible para sus alumnos, para que ellos construyan el conocimiento, lo co-construyan y lo repliquen cuando sea necesario. Los resultados encontrados apoyan la argumentación de los autores al demostrar que un lenguaje adecuado influye en la participación del alumnado.

López y Villalobos (2010) y Polanco (2005) consideran además que es primordial que el profesor vaya de lo simple a lo complejo en los contenidos de la clase, y que dichos contenidos se adecuen al tiempo y espacio en que se está aprendiendo, asegurándose que todos los alumnos hayan entendido. Esto se observó en el discurso de los participantes cuando el alumno daba una respuesta a una pregunta y el profesor contestaba que estaba bien, pero que lo retomaría más adelante, entendiendo que el profesor sigue una secuencia y estructura al dar sus clases y no se desvía del tema principal, pues tal como lo dice Adams (2012), los alumnos comprenden con facilidad los temas cuando los profesores planean sus sesiones, son coherentes con los objetivos de clase, y utilizan los métodos adecuados para enseñar.

La instrucción que da el profesor y el cómo lo hace, tendrá impacto en la motivación del estudiante; si es positivo, entonces los alumnos atenderán, escucharán, participarán y se involucrarán en la clase y en su aprendizaje; de lo contrario, no contestarán a las preguntas y no harán participaciones voluntarias (Fernandes y Alves, 2012). La presente investigación dio cuenta de esto cuando el profesor explicaba los contenidos de la materia de modo teórico y conceptual, y los alumnos respondían a las preguntas que se les formulaban de manera correcta y participaban sin que se les solicitara, utilizando el mismo lenguaje, lo que refleja que construyen nuevos conocimientos con esta manera de explicar. Asimismo, Bolarin et al. (2015) consideran que los alumnos se motivan si los contenidos que el profesor transmite son relevantes con la clase, por lo que se observa en los resultados que el profesor tiene un repertorio amplio de 
habilidades comunicativas para expresar y compartir los contenidos del currículo.

El alumno se vio motivado en la clase de medicina porque contestaba correctamente las preguntas que el profesor le planteaba, con la intención de compartir el conocimiento que tenía con sus demás compañeros, haciendo una aportación profunda cuando era una participación voluntaria, contrario a cuando era establecida. Sin embargo, el alumno no cuestionó al profesor, no hizo preguntas para que éste aclarara sus dudas acerca de un tema, ni mucho menos reflexionó respecto a los temas de clase y sus respuestas, es decir, no utilizó su propia experiencia como referente crítico. Ello se debe a que la estructura de la clase se torna monótona por su rigidez, falta de novedad y didáctica de los maestros; los alumnos piensan que las clases deben llevarse a cabo de manera tradicional, con el profesor al frente de la clase exponiendo el conocimiento, por lo que no tienen mayor interés en participar ni crear una reflexión o crítica ante lo que se estaba enseñando (Adams, 2012).

Ahora bien, para responder a la pregunta de investigación de cómo incide la retroalimentación afectiva del profesor en la motivación extrínseca del alumno, es decir, en su participación, se observó que la retroalimentación afectiva que el profesor ofrece se caracteriza por apreciar las respuestas de los alumnos, esto es, cuando el alumno participaba, enseguida el profesor repetía la respuesta correcta del alumno como una forma de aceptación. Además, el profesor estimulaba a los alumnos para que complementaran su respuesta con la finalidad de que quedara claro el aterrizaje del conocimiento en cuanto al tema de la clase. Coincidiendo con Savovic, Ubovic y Radenovic (2018), al explicar que quien está al frente de un grupo puede comunicarse de manera efectiva con los otros por medio de comentarios constructivos, positivos, de reconocimiento y motivacionales para lograr que el aprendizaje sea continuo y duradero, no sólo momentáneo, proponiendo que la retroalimentación afectiva del profesor permite una estructura de clase flexible y abierta a la participación del alumnado. De acuerdo con Héctor (2012), el discurso cognitivo no será suficiente para que el alumno se sienta confiado de sus conocimientos y poder participar en clase, por lo que debe implementarse el apoyo motivacional y afectivo en el aprendizaje. Es por ello que se acepta la hipótesis de investigación de que la retroalimentación afectiva incide en la motivación extrínseca del alumno, es decir, que cuando el profesor reconoce y alienta al alumno, éste participa de manera voluntaria.

Por último, se afirma que la motivación es un factor predisponente en el proceso de enseñanza-aprendizaje.
Si el alumno es motivado, existe mayor posibilidad de que se involucre en su aprendizaje y se reduce la posibilidad de que tenga un fracaso escolar, además de que el profesor genera condiciones de apoyo y seguridad (Héctor, 2012; Polanco, 2005). No obstante, en la motivación extrínseca los alumnos tienen que afrontar el reconocimiento de otro para estar motivados, aprender y obtener buenas notas; cuando lo alumnos no cumplen con las expectativas que el otro tiene de él, se torna vulnerable a fracasar y disminuir en su rendimiento académico, teniendo como consecuencia una desmotivación por aprender y continuar sus estudios; esto hace relevante la manera en que el profesor retroalimenta las aportaciones del alumnado, generando condiciones de apertura y participación al demostrar afecto e interés en el salón de clases, volviéndose el andamio afectivo para que el alumno pueda involucrarse en su propio conocimiento.

\section{CONCLUSIONES}

La psicología educativa se enfoca en desarrollar y mejorar las capacidades de las personas involucradas en las instituciones educativas, es decir, de profesores-alumnos (Pérez, 2019). Cada uno de ellos cuenta con habilidades que ayudan a que este proceso sea o no exitoso; las conductas de uno van a influir en el otro, y por tanto en el proceso de enseñar y aprender. De este modo, se concluye que si los expertos son conscientes del discurso utilizado durante las clases pueden conducir a sus alumnos a tener mayor participación en la construcción del conocimiento, desarrollando la motivación extrínseca en ellos. No se debe olvidar que los alumnos aprenden y se apropian de las formas de habla y modos de discurso específicos del escenario sociocultural en que están inmersos (Prados et al., 2010), por lo que construyen nuevas formas de comprender y de explicar la realidad conforme se va construyendo el discurso en el aula.

A su vez, Héctor (2012) argumenta que el alumno motivado logra formular preguntas y cuestionamientos al profesor para confirmar un conocimiento o descubrir algo nuevo; si el profesor observa el interés del alumno, le reconoce su valentía por preguntar, y el alumno se siente seguro de volver a cuestionar sobre el tema de clase. Del mismo modo, Seder et al. (2018) consideran que el alumno participará en la clase sólo si las estrategias del profesor le agradan. Además, Barrios (2008), González (2005) y Mayoral y Castelló (2015) identificaron que la retroalimentación afectiva, como el reconocimiento y el agradecimiento, fomentan la motivación extrínseca del alumno, puesto que éste se involucra en la clase con su participación activa. 
Para investigaciones futuras se recomienda, en primera instancia, que se amplíen las sesiones de grabación para tener mayor consistencia en el dato, ya que en este estudio sólo se hizo una observación. Es necesario aclarar que la cámara de video no fue limitante para que los alumnos actuaran de manera natural en su clase, por lo cual la observación con instrumentos de grabación es adecuada (Tronchoni, Izquierdo y Anguera, 2018). El dato que se obtuvo fue científico; de acuerdo con Anguera (2003), la observación natural permite al investigador observar a su población en un contexto natural - en este caso, en la clase de medicina-y recabar información acerca de lo que está sucediendo, pues no se manipulan el ambiente ni otra variable.

Además se invita a estudiar y ampliar los hallazgos de las categorías que presentaron una frecuencia y porcentaje bajo, de acuerdo con el marco teórico, ya que en este estudio no fueron retomadas; las categorías fueron pregunta dirigida al profesor (PreDiP) y solicitud de participación (SolP) por parte del alumno, y reconocimiento (RecPro) y agradecimiento (AgraPro) a la participación del alumno por parte del profesor. A partir de los resultados se dedujo que el estudiante no se sintió seguro para expresar alguna duda y cuestionar al maestro respecto a lo que él mismo entendía, y no solicitó un turno de habla porque no conocía la respuesta, o porque no quería equivocarse. Mientras que el profesor no reconoció ni agradeció el esfuerzo del estudiante por participar, estuviera o no en lo correcto, el estudiante podría haber esperado esta forma de retroalimentación para sentirse seguro durante su intervención en la clase. Sería valioso indagar la manera en que se puede motivar a los participantes a mantener un diálogo donde se promueva la atención y consideración del turno del otro durante sus participaciones en el discurso en el aula.

Por último, se recomienda agregar la participación y vivencia del profesor, indagando acerca de las acciones o situaciones que él considera que motivan al alumno, cuáles le han resultado eficientes y cuáles considera mejorar e implementar. Además, proponer un acercamiento con el alumno para conocer su opinión y experiencia respecto a las acciones del profesor que son o no motivantes para él. Esto podría realizarse por medio de un instrumento o inventario que permita medir el tema de interés, o en su defecto con una guía de preguntas elaboradas a partir del marco teórico y las referencias encontradas.

\section{Referencias}

Adams, J. A. (2012). Valoración de estudiantes universitarios sobre las didácticas significativas para su aprendizaje. Revista Iberoamericana de Psicología: Ciencia y Tecnología, 5(2), 31-39. Recuperado de https://reviberopsicologia. ibero.edu.co/article/view/243/211

Alemán-Marichal, B., Navarro-de-Armas, O., Suárez-Díaz, R. M., Izquierdo-Barceló, Y., \& Encinas-Alemán, T. (2018). La motivación en el contexto del proceso enseñanza aprendizaje en carreras de las Ciencias Médicas. Revista Médica Electrónica, 40(4), 1257-1270. Recuperado de http://www.revmedicaelectronica.sld.cu/index.php/ rme/article/view/2307

Anguera, M. T. (2003). La observación. En C. Moreno (ed.), Evaluación psicológica. Concepto, proceso y aplicación en las áreas del desarrollo y de la inteligencia, 271-308. Madrid: Sanz y Torres. http://www.observesport.com/ desktop/images/docu/gh87qq4t.pdf

Anguera, M. T., \& Blanco-Villaseñor, A. (2006). ¿Cómo se lleva a cabo un registro observacional? Butlletí La Recerca, 4, 1-7. https://www.ub.edu/idp/web/sites/default/files/ fitxes/ficha4-cast.pdf

Anguera, M. T., Blanco-Villaseñor, A., Losada, J. L., \& Portell, M. (2018). Pautas para elaborar trabajos que utilizan la metodología observacional. Anuario de Psicología, 48, 9-17. https://doi.org/10.1016/j.anpsic.2018.02.001

Anguera, M. T., Magnusson, M. S., \& Jonsson, G. K. (2007). Instrumentos no estándar. Avances en Medición, 5(1), 63-82. https://www.humanas.unal.edu.co/lab psicometria/application/files/8016/0463/3543/ Vol_5._Instrumentos_No_Estndar___Planteamiento_ Desarrollo_Y_Posibilidades.p̄df

Barrios, E. (2008). Categorización del feedback suministrado a futuros profesores y maestras durante las Prácticas de Enseñanza en el contexto de una modalidad interactiva de escritura de ensayos sobre diarios de prácticas. En C. Marcelo (presidencia). I Congreso Internacional sobre Profesorado Principiante e Inserción Profesional a la Docencia, Facultad de Ciencias de la Educación: Sevilla. https://www.yumpu.com/es/ document/view/14485818/m-elvira-barrios-espinosacategorizacion-del-prometeo

Black, P., \& Wiliam, D. (2004). The formative purpose: Assessment must first promote learning. En M. Wilson (ed.), Towards coherence between classroom assessment and accountability. Yearbook of the National Society for the Study of Education, 103(2), 20-50. https://doi. org/10.1111/j.1744-7984.2004.tb00047.x

Bolarin, M. J., Porto, M., Martínez, N., \& Méndez, R. (2015). Dimensiones de la motivación desde la perspectiva del alumnado: 20 años de investigación. Psicología Em Estudo, 20(4), 599-610. https://doi.org/10.4025/ psicolestud.v20i4.28227

Boza-Carreño, A., \& De la O Toscano, M. (2012). Motivos, actitudes y estrategias de aprendizaje: Aprendizaje motivado en alumnos universitarios. Profesorado. Revista de Currículum y Formación de Profesorado, 16(1), 125-142. 
http://www.redalyc.org/articulo.oa?id=56724377008

Bruno, I., \& Santos, L. (2010). Written comments as a form of feedback. Studies in Educational Evaluation, 36(113), 111-120. https://doi.org/10.1016/j.stueduc.2010.12. Casasempere, A. [MAXQDA VERBI] (28 de noviembre de 2017). Introducción a la investigación cualitativa con MAXQDA (Spanish) [Video YouTube]. https://www. youtube.com/watch?v=emwE89gpX4Y

Echeverría, J., Pacenza, M. I., \& Urquijo, S. (2011). Participación de estudiantes en actividades académicas: Motivación y nivel de información. PSIENCIA. Revista Latinoamericana de Ciencia Psicológica, 3(2), 82-93. Recuperado de http:// www.redalyc.org/articulo.oa?id=333127105003

Fernandes, D. C., \& Alves, M. (2012). Evaluación de la motivación académica y la ansiedad escolar y posibles relaciones entre ellas. Psico-USF, 17(3), 447-455. Recuperado de https://www.redalyc.org/pdf/4010/401036091011. pdf

García, M. E., \& Cruz, M. L. (2016). El alumno motivado: Un análisis empírico de los factores motivadores intrínsecos y extrínsecos en el aula de inglés. Revista académica evaluada por pares y de acceso abierto (90), 72-93. http:// hdl.handle.net/11441/59646

González, A. (2005). Motivación académica: Teoría, aplicación y evaluación. España: Psicología Pirámide.Héctor, E. F. (2012). La motivación como sustento indispensable del aprendizaje en los estudiantes universitarios. Pedagogía universitaria, 17(4), 13-27. https://www.academia. edu/18920231/La_motivaci\%C3\%B3n_como_ sustento_indispensable_del_aprendizaje_en_los_ estudiantes_universitarios

Jiménez,F.(2014).Usodelfeedbackcomoestrategiadeevaluación: Aportes desde un enfoque socioconstructivista. Revista Electrónica "Actualidades Investigativas en Educación", 15(1), 1-24. https://doi.org/10.15517/aie.v15i1.17633

López, A. A. (2010). La evaluación formativa en la enseñanza y aprendizaje del inglés. Voces y Silencios: Revista Latinoamericana de Educación, 1(2), 111-124. https:// revistas.uniandes.edu.co/doi/pdf/10.18175/ vys1.2.2010.01

López, S. P., \& Villalobos, N. L. (2010). Construcciones metodológicas de profesores en cursos electivos universitarios. Revista Iberoamericana de Psicología: Ciencia y Tecnología, 3(2), 17-26. https:// reviberopsicologia.ibero.edu.co/article/view/rip.3202

Lundgren, M., \& Lökholm K. (2006). Motivationshöjande samtal i skolan. Studentlitteratur. Lund: Studentlitteratur.

Mayoral S. P., \& Castelló, M. (2015). Profesorado novel y feedback del tutor. Un estudio de casos. Revista de Currículum y Formación de Profesorado, 19(1), 346-362. https://recyt. fecyt.es/index.php/profesorado/article/view/41045

Ortiz, D. (2015). El constructivismo como teoría y método de enseñanza. Sophia, Colección de Filosofía de la
Educación (19), 93-110. https://doi.org/10.17163/soph. n19.2015.04

Pérez, G. C. (2019). Aportes desde la psicología educativa para el desarrollo de los valores y ética profesional. Revista de Investigación Psicológica (21), 11-37. http://www.scielo. org.bo/pdf/rip/n21/n21_a03.pdf

Polanco, A. (2005). La motivación en los estudiantes universitarios. Revista electrónica Actualidades Investigativas en Educación, 5(2), 1-13. http://www. redalyc.org/articulo.oa?id=44750219

Prados-Gallardo, M., Cubero-Pérez, M., \& De la Mata-Benítez, M. L. (2010). ¿Mediante qué estructuras interactivas se relacionan profesorado y alumnado en las aulas universitarias? Electronic journal of research in Educational Psychology, 8(1), 163-194. http://www. redalyc.org/articulo.oa?id=293121995008

Robles, P., \& Rojas, M. D. C. (2015). La validación por juicio de expertos: dos investigaciones cualitativas en Lingüística aplicada. Revista Nebrija de Lingüística Aplicada, 18, 1-16. https://www.nebrija.com/revista-linguistica/lavalidacion-por-juicio-de-expertos-dos-investigacionescualitativas-en-linguistica-aplicada.html

Ruiz, E., Méndez, V., \& Cruz, J. L. (2017). Análisis secuencial discursivo de una práctica de enfermería utilizando SDIS-GSEQ: la relación profesor-alumno. CPUE, Revista de Investigación Educativa (24), 154-175. Recuperado de http://www.redalyc.org/articulo.oa?id=283149560008

Savovic, B., Ubovic, M., \& Radenovic, S. (2018). Analysis of the motivation speech held by the national team coach before the final FIFA U-20 world cup match. Physical Culture, 72(1), 102-110. http://dx.doi.org/10.5937/ fizkul1801102S

Seder, A., Castell, R., \& Moreno, F. (2018). ¿Un cambio de metodología que aumente la satisfacción y motivación del estudiante favorece su aprendizaje? Experiencias en el aula. Actas de las Jenui, 3, 335-342. https://www.uv.es/ grimo/publications/jenui2018.pdf

Sociedad Mexicana de Psicología (2010). Código Ético del Psicólogo. México: Trillas.

Tronchoni, H., Izquierdo, C., \& Anguera, M. A. (2018). Interacción participativa en las clases magistrales: Fundamentación y construcción de un instrumento de observación. Publicaciones, 48(1), 81-108. https://doi.org/10.30827/ publicaciones.v48i1.7331

Urra, E., Muñoz, A., \& Peña, J. (2013). El análisis del discurso como perspectiva metodológica para investigadores de salud. Enfermería Universitaria, 10(2), 50-57. https://doi. org/10.1016/S1665-7063(13)72629-0

Valenzuela, J., Muñoz, C., Silva-Peña, I., Gómez, V., \& Precht, A. (2018). Motivación escolar: Claves para la formación motivacional de futuros docentes. Estudios Pedagógicos, 41(1), 351-361. https://doi.org/10.4067/S071807052015000100021 


\section{Meta-Análisis del Artículo}




\section{Dimensión Cuantitativa}

\section{Perfil de Evaluación entre pares}
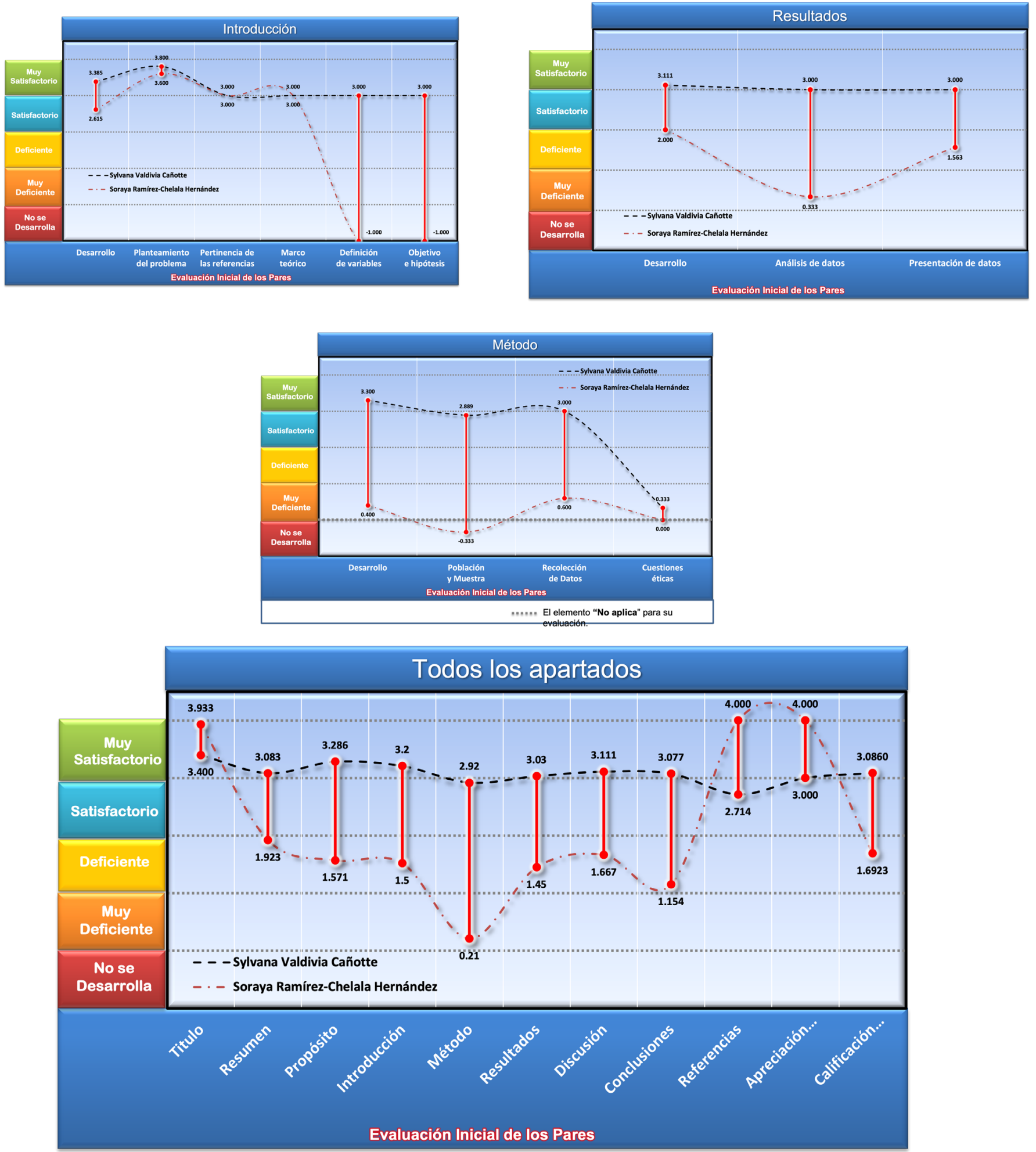
Artículo Empirico |Análisis de la motivación extrínseca y la retroalimentación...| García-Corona, et al. | e08012022376

\section{Índice de Concordancia}

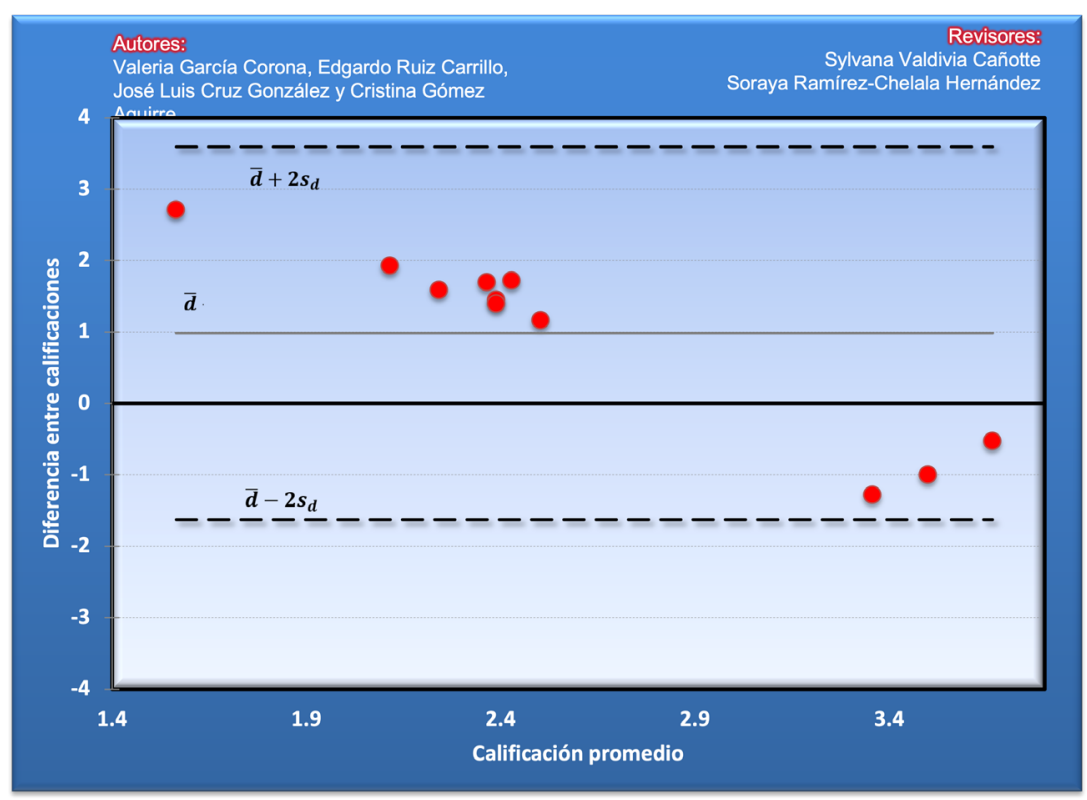

\section{Índice de Acuerdo}

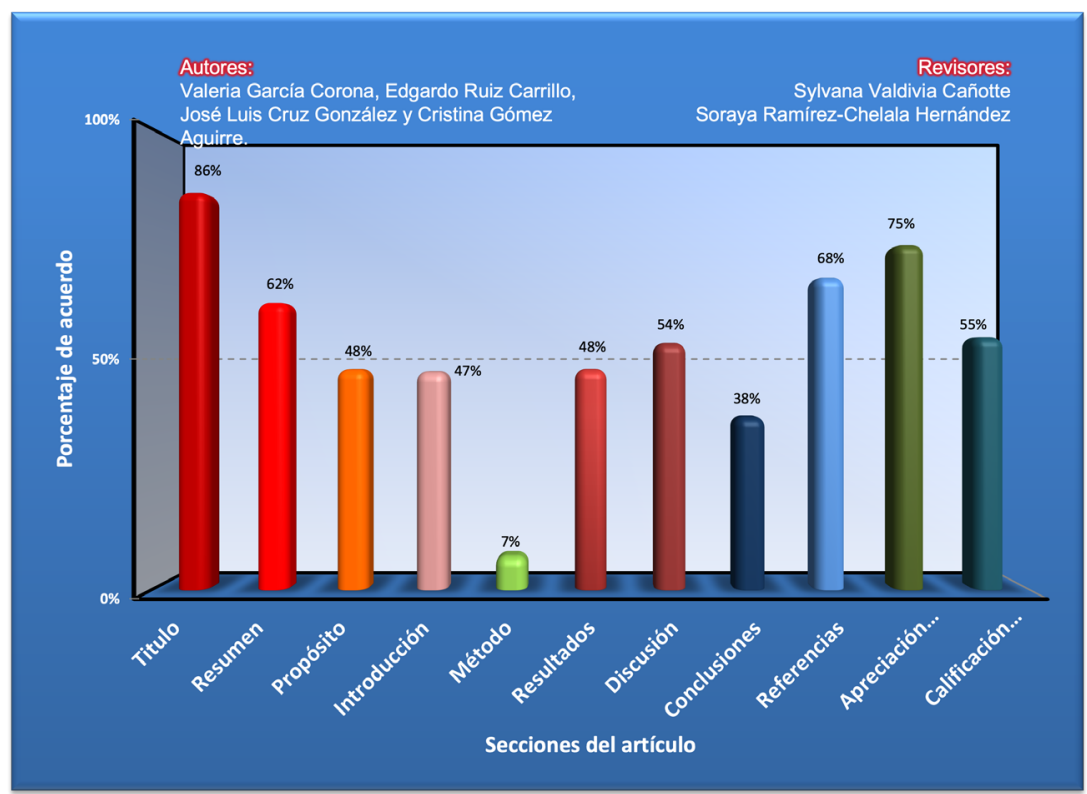




\begin{tabular}{|c|c|}
\hline Revisor 1 & Revisor 2 \\
\hline Soraya Ramírez-Chelala Hernández & Sylvana Valdivia Cañotte \\
\hline \multicolumn{2}{|c|}{ Título/Autoría } \\
\hline Sin comentarios & $\begin{array}{l}\text { Podría considerarse incluir "retroalimentación afectiva" } \\
\text { en tanto se desarrolla en la investigación y constituye una } \\
\text { palabra clave. }\end{array}$ \\
\hline \multicolumn{2}{|c|}{ Resumen } \\
\hline $\begin{array}{l}\text { De los puntos } 3 \text { al } 6 \text {, sí están, aunque no se separan por } \\
\text { párrafos. Debe indicarse incluirse el tipo de investiga- } \\
\text { ción que se realizó, el diseño y el tipo de muestra que se } \\
\text { utilizó. Debe incluirse, de manera clara, la pregunta de } \\
\text { investigación. }\end{array}$ & Agregar una palabra clave. \\
\hline \multicolumn{2}{|c|}{ Próposito del Estudio } \\
\hline $\begin{array}{l}\text { Hablan de la retroalimentación afectiva, pero no aco- } \\
\tan \text { a qué se refieren con ella. }\end{array}$ & $\begin{array}{l}\text { Se evidencia coherencia entre el propósito y la estructura } \\
\text { del estudio. }\end{array}$ \\
\hline \multicolumn{2}{|c|}{ Introducción } \\
\hline $\begin{array}{l}\text { El punto } 9.9 \text { no se cumple porque no hay ni hipótesis ni } \\
\text { pregunta de investigación. }\end{array}$ & $\begin{array}{l}\text { Se recomienda centrarse en estudios del ámbito de la } \\
\text { medicina en educación superior. Revisar la lista de } \\
\text { referencias; algunas no se encuentran en el texto o el } \\
\text { nombre de los autores no están escritos de la misma } \\
\text { forma. }\end{array}$ \\
\hline \multicolumn{2}{|c|}{ Método } \\
\hline $\begin{array}{l}\text { Están incorrectos los criterios de inclusión / exclusión. } \\
\text { Por ejemplo, en el apartado de participantes ponen: } \\
\text { Los criterios de inclusión para la profesora, más parece } \\
\text { la descripción de la profesora que los criterios de in- } \\
\text { clusión y exclusión del sujeto que sería el profesor. No } \\
\text { acotan de manera concisa, los criterios de inclusión y } \\
\text { exclusión de los sujetos alumnos No indican el diseño } \\
\text { de la investigación }\end{array}$ & $\begin{array}{l}\text { Se sugiere hacer precisiones al apartado correspondiente a } \\
\text { consideraciones éticas. }\end{array}$ \\
\hline \multicolumn{2}{|c|}{ Resultados } \\
\hline $\begin{array}{l}\text { 21.1, } 21.2,22.3,22.4,23.1 \text { y } 23.4 \text { se ponen como no se de- } \\
\text { sarrolló porque no hay hipótesis/preguntas/objetivos En } \\
\text { el } 21.3 \text { falta la relación entre gráfica } 1 \text { y } 2\end{array}$ & $\begin{array}{l}\text { Los resultados son claros y se complementan con las ta- } \\
\text { blas y gráficos. }\end{array}$ \\
\hline
\end{tabular}


Artículo Empírico |Análisis de la motivación extrínseca y la retroalimentación...| García-Corona, et al. | e08012022376

\begin{tabular}{|c|c|}
\hline Revisor 1 & Revisor 2 \\
\hline \multicolumn{2}{|c|}{ Discusión } \\
\hline $\begin{array}{l}\text { 25.4, se ponen como no se desarrolló porque no hay } \\
\text { hipótesis/preguntas/objetivos }\end{array}$ & $\begin{array}{l}\text { Se evidencian interpretaciones suficientes y coherentes } \\
\text { con lo que se deseaba indagar. }\end{array}$ \\
\hline \multicolumn{2}{|c|}{ Conclusiones } \\
\hline $\begin{array}{l}\text { 27.3, 27.4, se ponen como no se desarrolló porque no hay } \\
\text { hipótesis/preguntas/objetivos }\end{array}$ & $\begin{array}{l}\text { Se podría profundizar en los siguientes pasos o la reco- } \\
\text { mendación para futuras investigaciones. }\end{array}$ \\
\hline \multicolumn{2}{|c|}{ Referencias } \\
\hline Sin comentarios. & $\begin{array}{l}\text { Verificar que todas las vitas estén en la lista de referencias } \\
\text { y viceversa. Verificar el apellido de algunos autores y uni- } \\
\text { formizar su correcta escritura en el texto. }\end{array}$ \\
\hline
\end{tabular}




\section{Historia del Proceso}

\section{EDITORIAL}

